\title{
Paradoxe "Low-Flow-Low-Gradient"-Aortenklappenstenose
}

\section{Ein diagnostisches Dilemma}

\author{
Bei atypischen "Low-Flow-Low-Gradient-Aortenklappenstenose" ist \\ die Diagnose schwierig, vor allem dann, wenn die linksventrikuläre \\ (LV-)Funktion paradoxerweise nicht beeinträchtigt ist.
}

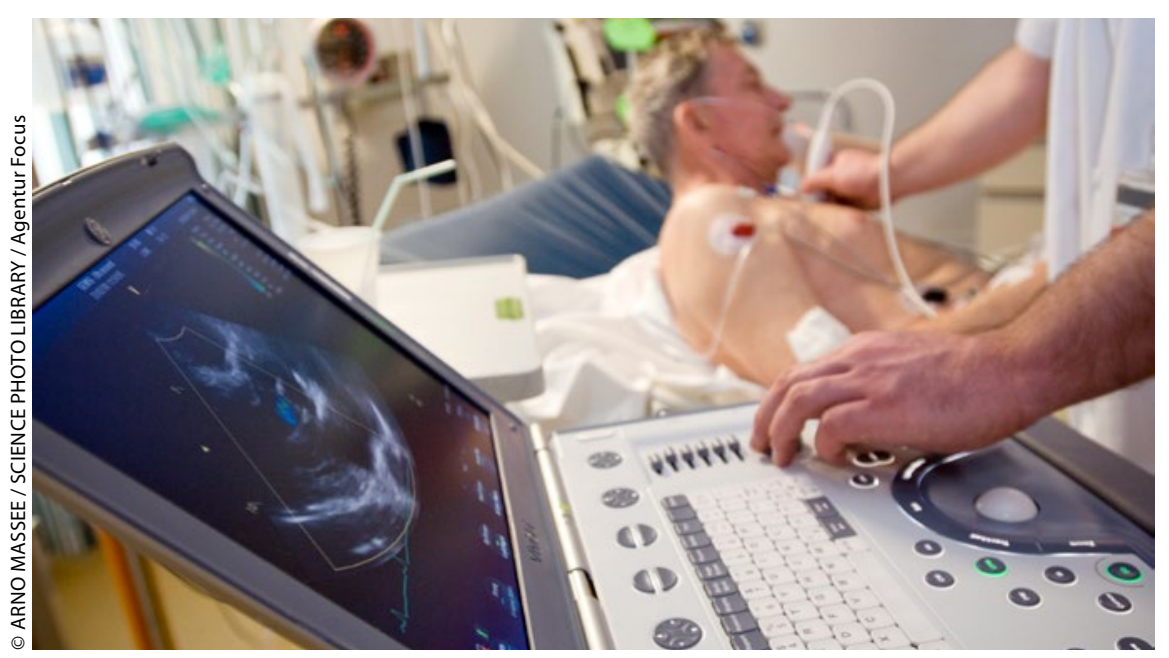

Die eingeschränkte LV-Funktion mit vermindertem Schlagvolumen bei einer LowFlow-Gradient-Aortenstenose ist bei guten Schallbedingungen kaum zu übersehen.

$\mathrm{D}$ ie Diagnose der hochgradigen Aortenstenose ist einfach: Maximale Jetgeschwindigkeit $>4 \mathrm{~m} /$ sec, mittlerer Druckgradient $>40 \mathrm{mmHg}$, Klappenöffnungsfläche $<1 \mathrm{~cm}^{2}$, indizierte Klappenöffnungsfläche $<0,6 \mathrm{~cm}^{2} / \mathrm{m}^{2}$ und ,velocity ratio“ $V_{\max } / \mathrm{VLVOT}>4$.

Schwieriger ist die Situation bei atypischen Aortenklappenstenosen, bei denen die Klappenöffnungsfläche zwar $<1 \mathrm{~cm}^{2}$, aber die maximale Jetgeschwindigkeit $<4$ $\mathrm{m} / \mathrm{sec}$ bzw. der mittlerer Druckgradient $<40 \mathrm{mmHg}$ beträgt. Die Frage hier: Handelt es sich um eine hochgradige Aortenklappenstenose oder um eine Pseudostenose? Wer nur Gradienten messe, werde solche Patienten übersehen, sagte Prof. Gregor Simonis aus Dresden. Daher sei die zusätzliche Bestimmung der Aortenklappenöffnungsfläche obligat. Die klassische Low-Flow-Low-Gradient-Aortenklappenstenose bei eingeschränkter LVFunktion mit vermindertem Schlagvolu- men ist bei guten Schallbedingungen kaum zu übersehen. In unklaren Fällen empfiehlt sich ein Dobutamin-StressEcho. Wenn die Öffnungsfläche $<1 \mathrm{~cm}^{2}$ bleibt und der Gradient steigt, liegt eine echte hochgradige Aortenklappenstenose vor. Bei stabilem Gradienten und $\mathrm{Zu}$ nahme der Öffnungsfläche $>1 \mathrm{~cm}^{2}$ liegt eine Pseudostenose vor, die keiner Therapie bedarf.

Paradox ist die Situation, wenn sich eine Low-Flow-Low-Gradient-Aortenklappenstenose bei Patienten mit normaler LV-Funktion zeigt. Bei diesen Patienten ist der linke Ventrikel hypertrophiert und es finden sich Hinweise auf eine diastolische Funktionsstörung, also das Bild einer diastolischen Herzinsuffizienz. Die LV-Volumina sind klein und somit auch das Schlagvolumen, das $<35 \mathrm{ml} / \mathrm{m}^{2}$ beträgt. „Die EF wirkt zwar noch normal, aber der longitudinale Strain ist in der Regel bereits abnormal“, so Simonis.

\section{Eine ziemlich neue Diagnose}

Bei der paradoxen Low-Flow-Low-Gradient-Aortenklappenstenose handelt es sich um eine "neue“ Diagnose. Es gibt kaum wissenschaftliche, vor allem keine prospektiven Daten. In einer Analyse von 512 Patienten mit Aortenklappenöffnungsflächen-Index $<0,6 \mathrm{~cm}^{2} / \mathrm{m}^{2}$ und einer EF $>50 \%$ war diese Stenose bei Frauen häufiger als bei Männern und der Gradient und die EF etwas niedriger als mit normalem Fluss. Auch war die 3-Jahres-Überlebensrate mit 76\% im Vergleich zu $86 \%$ schlechter. Doch das schlechtere Überleben resultiere vorwiegend aus dem Krankheitsbild, das seltenen korrekt diagnostiziert werde, und dem Klappenersatz, der oft nicht erfolge, so Simonis; auch diese Patienten profitierten eindeutig vom Klappenersatz, wenn sie symptomatisch seien.

Doch ehe man die Diagnose „paradoxe Low-Flow-Low-Gradient-Aortenklappenstenose" stellt, müssen Messfehler im Echo ausgeschlossen sein, vor allem bei der LVOT-Messung. Messungen von apikal sind oft ungenau, sodass im Zweifelsfall immer eine TEE erfolgen sollte. Bei Vorhofflimmern muss man viele Zyklen messen und dann mitteln. Zudem sollte man, da es sich meist um kleine Patienten handelt, die Öffnungsfläche immer auf die Körperoberfläche indexieren. Bei grenzwertigen Befunden kann ein Dobutamin-Stress-Echo weiterhelfen, wobei die Aussagekraft nur bei Diagnosebestätigung zuverlässig ist. Auch das CT bzw. die Quantifizierung des Aortenklappenkalks kann hilfreich sein. Da ein unzureichend eingestellter Hypertonus das Schlagvolumen weiter reduziere, sollte nach optimaler Blutdruckeinstellung bei bestehender Symptomatik immer eine Reevaluierung der Echo-Befunde erfolgen, ehe die Indikation zum Klappenersatz gestellt werde, so Simonis. Spricht aber alles für eine echte Low-Flow-LowGradient-Aortenklappenstenose, ist ein Klappenersatz indiziert. Peter Stiefelhagen 